\title{
Efeito de um programa misto de intervenção nutricional e exercício físico sobre a composição corporal e os hábitos alimentares de mulheres obesas em climatério
}

\author{
The effects of a mixed program of nutritional \\ intervention and physical exercise on body composition \\ and feeding habits of obese climacteric women
}

Rita de Cássia de Assunção MONTEIRO'

Priscila Trapp Abbes RIETHER ${ }^{2}$

Roberto Carlos BURINI ${ }^{3}$

\section{R E S U M O}

\section{Objetivo}

Foi avaliar o efeito de programa misto de intervenção nutricional e exercício físico sobre a composição corporal e hábitos alimentares de mulheres obesas em climatério.

\section{Métodos}

Trabalhou-se com 2 grupos de 15 pessoas por 40 semanas: Grupo Dieta (intervenção nutricional) e Grupo Exercício (intervenção nutricional e exercício).

\section{Resultado}

As reduções do peso (-2,3kg para Grupo Dieta e -5,3kg para o Grupo Exercício) e da circunferência da cintura (-4,8cm para Grupo Dieta e -7,6cm para Grupo Exercício), foram maiores para o Grupo Exercício. Foi verificada evolução positiva na classificação do Índice de Massa Corporal para ambos os grupos, sendo que o Grupo Exercício respondeu melhor ao tratamento. O padrão alimentar foi considerado monótono e com baixo consumo de alimentos regionais.

\footnotetext{
1 Curso de Nutrição, Centro Universitário Nilton Lins. Av. Prof. Nilton Lins, 3259, Parque das Laranjeiras, 69058-040, Manaus, AM, Brasil. Correspondência para/Correspondence to: R.C.A. MONTEIRO.E-mail: rita-monteiro@uol.com.br

2 Faculdade de Educação Física, Universidade Federal do Amazonas. Av. Gen. Rodrigo O. Jordão Ramos, 3000, Campus Universitário, 69077-000, Manaus, AM, Brasil.

3 Departamento de Clínica Médica, Faculdade de Medicina, Universidade Estadual Paulista. Botucatu, SP, Brasil.
} 


\section{Conclusão}

O programa foi efetivo para perda de peso, em maior intensidade na presença de exercício. A educação alimentar proposta foi capaz de acarretar mudanças nos hábitos alimentares.

Termos de indexação: obesidade, climatério, exercício físico, dieta, educação nutricional.

\section{A B S T R A C T}

\section{Objective}

The effects of a mixed program of nutrition intervention and physical exercise on body composition and feeding habits were evaluated in obese climacteric women.

\section{Methods}

Thirty participants were randomly assigned in 2 groups during 40 weeks: Diet Group (nutritional intervention) and Exercise Group (nutritional intervention and exercise).

\section{Results}

Weight reductions (Diet Group -2,3kg/Exercise Group -5,3kg) and waist circunference (Diet Group -4,8cm/ Exercise Group -7,6cm) were greater in Exercise Group. A positive evolution was noticed in the Body Mass Index in both groups, with Exercise Group having better answer to treatment. It was observed a monotonous feeding pattern and low compliance to the consumption of regional typical foods.

\section{Conclusion}

We concluded that the program was effective for weight loss at a higher intensity in the presence of exercise and the feeding education was able to modify the feeding habits.

Index terms: obesity, climacterium, physical exercises, nutritional education, diet.

\section{N T R O D U Ç Ã O}

Considerada atualmente como epidemia, a obesidade vem crescendo de forma alarmante tanto nos países desenvolvidos como naqueles em desenvolvimento, acarretando enormes prejuízos à sociedade.

A obesidade é uma doença crônica que se caracteriza pelo acúmulo excessivo de gordura num nível tal que passa a comprometer a saúde do indivíduo, pois está associada ao aumento dos riscos para o acometimento de doenças cardíacas, aterosclerose, hipertensão arterial, hiperlipidemias, diabetes, osteoartrites, patologias biliares e vários tipos de câncer.

Do ponto de vista epidemiológico, observa-se que a obesidade ocorre principalmente em indivíduos do sexo feminino, de classe social menos favorecida e sua incidência parece aumentar com a idade'.

A alimentação desequilibrada e o sedentarismo constituem os fatores mais freqüentemente apontados como determinantes do súbito aumento dos casos de obesidade entre as populações, representando, portanto, variáveis importantes a serem exploradas, especialmente em alguns grupos mais vulneráveis, como mulheres na pós-menopausa².

Cerca de $60 \%$ das mulheres na idade da menopausa apresentam um rápido ganho de peso entre $2,5 \mathrm{~kg}$ e 5,0kg, cuja etiologia permanece ainda desconhecida ${ }^{3}$. Em sociedades industrializadas geralmente ocorre um incremento do peso corporal com a idade. No entanto, o término do ciclo menstrual não afeta a ingestão de alimentos 
e reduz pouco a Taxa de Metabolismo Basal; logo, uma parcela do peso ganho nesta fase, pode ser atribuído à simples redução da atividade física².

A obesidade perimenopausal vem atraindo muita atenção por parte dos pesquisadores, não só porque afeta um número considerável de mulheres, mas também devido sua forte correlação com o aumento dos riscos de morbimortalidade nessa idade, com destaque para as doenças cardiovasculares. Uma hipótese para esse fenômeno seria o decréscimo da função ovariana e a conseqüente deficiência de estrogênio, que parece interferir de maneira desfavorável no perfil de lipoproteínas plasmáticas e na distribuição do tecido adiposo ${ }^{3}$.

Com relação às propostas de controle da obesidade, a maioria parece conduzir ao equilíbrio da ingestão energética somado ao aumento da atividade física, sem, no entanto haver consenso sobre os tipos, duração e níveis de exercício e de dieta mais adequados às diversas situações. Nesse contexto, a principal etapa do tratamento da obesidade deve consistir numa reestruturação do comportamento do indivíduo, em busca de um estilo de vida saudável ${ }^{1,4}$.

A comunidade científica vem acumulando evidências para demonstrar a relação entre dieta e saúde, especialmente na prevenção e no controle das enfermidades crônicas não-transmissíveis, incluindo a obesidade. Apesar dos esforços, essa interação permanece pouco compreendida, talvez, pelas dificuldades inerentes ao estudo e à aferição do consumo alimentar humano com suficiente confiança e precisão.

Está documentado que altos níveis de ingestão de gordura e calorias associam-se fortemente ao excesso de peso corporal, especialmente com aumento do tecido adiposo. Além disso, alguns estudos demonstraram, que a composição da dieta, especialmente o seu conteúdo em gordura, mais do que o consumo energético total, possui um importante papel no desenvolvimento da obesidade 5 . Portanto, o obeso precisa adquirir hábitos alimentares saudáveis, que contribuam para perda inicial e manutenção do peso corporal².
Inúmeros fatores influenciam a escolha qualitativa e quantitativa dos alimentos a serem ingeridos. Isto significa que os hábitos alimentares constituem o resultado das experiências apreendidas ao longo da vida; portanto, é possível, com algum esforço e técnicas eficazes de educação, reformular esses mesmos hábitos alimentares, no sentido de corrigir possíveis distúrbios nutricionais ${ }^{6}$.

Em relação ao exercício físico, há evidências científicas consistentes de que sua prática regular traz amplos benefícios para a saúde física e mental ${ }^{2}$. A atividade física pode influenciar de maneira positiva a composição corporal por meio de vários mecanismos, pois promove o aumento do gasto energético total, equilíbrio na oxidação dos macronutrientes e preservação da massa magra. Os efeitos no metabolismo energético vão depender do tipo, da intensidade, da duração e da freqüência do exercício desempenhado 1,2,4.

Segundo Mundim4, toda proposta terapêutica para a obesidade deve ser realista, maleável, com duração indeterminada e ter como meta principal uma melhor qualidade de vida, com ou sem redução de peso.

Nesse contexto, considerando a obesidade uma enfermidade complexa, que parece afetar uma ampla faixa da população, gerando graves conseqüências, o presente trabalho teve como objetivo avaliar o efeito de um programa de intervenção nutricional, acompanhado de exercício físico, sobre o perfil antropométrico e os hábitos alimentares de indivíduos obesos do sexo feminino no período do climatério.

\section{CASUÍSTICA E MÉTODOS}

Os indivíduos que compuseram a amostra estavam regularmente matriculados no projeto "Universidade na Terceira Idade Adulta" (Universidade Federal do Amazonas), que perfaz um total de 500 pessoas. Os critérios de inclusão foram: clínico - não apresentar nenhuma doença 
incapacitante para a prática de exercícios físicos; antropométrico - possuir Índice de Massa Corporal (IMC) igual ou superior a 30; sexo - ser do sexo feminino; menopausa - estar instalada há pelo menos 12 meses e disponibilidade - para participar das sessões de exercícios físicos e educação alimentar, bem como das avaliações antropométricas e nutricionais. Assim, das 500 pessoas matriculadas no projeto, 30 foram selecionadas para este estudo.

No início da pesquisa todos os indivíduos foram esclarecidos quanto aos objetivos e à metodologia a ser utilizada, sendo obtido o consentimento individual por escrito. O presente trabalho teve aprovação pelo Comitê de Ética em Pesquisas da Faculdade de Medicina da Universidade Estadual Paulista. Num segundo momento, as 30 mulheres selecionadas foram distribuídas de maneira inteiramente casual, formando dois grupos de 15 mulheres cada, conforme descrição a seguir: Grupo Dieta (GD) - somente intervenção nutricional e Grupo Exercício (GE) - intervenção nutricional acoplada a protocolo de exercício físico.

O período experimental teve duração de 40 semanas, sendo as avaliações antropométrica e de consumo alimentar realizadas no momento zero e ao final do período. A intervenção nutricional foi constituída de uma consulta inicial e 4 subseqüentes, de acompanhamento, a cada 10 semanas, além de um programa de educação alimentar, efetivado por meio de encontros quinzenais de 1 hora com cada grupo em separado, perfazendo um total de 20 encontros. O protocolo de exercício foi aplicado três vezes por semana, sendo uma sessão por dia e cada sessão com 60 minutos de duração.

A avaliação antropométrica foi realizada a partir da tomada de medidas de peso, altura, circunferências de cintura e de quadril conforme recomendação de especialistas ${ }^{7}$.

O Índice de Massa Corporal (IMC) foi calculado a partir das medidas de peso $(\mathrm{kg})$ e altura $(m)$, aplicando a fórmula: $I M C=P / A^{2}$. Empregou-se a classificação da 1a Convenção Latino-Americana para Consenso em Obesidade ${ }^{1}$ com o intuito de avaliar a magnitude do risco para a saúde em relação ao IMC. A relação cintura/quadril (C/Q), dividindo a circunferência da cintura pela do quadril, foi empregada na classificação de obesidade andróide, relação $C / Q>0,8$ e obesidade ginecóide, relação $C / Q<0,8$. A circunferência da cintura também foi interpretada de forma isolada1.

A avaliação do consumo alimentar foi realizada em nível individual, por meio do questionário de freqüência de consumo. 0 questionário foi composto de 58 alimentos com 9 possibilidades de resposta para o consumo: diário; 5, 4, 3, 2 ou 1 vez na semana; quinzenal; mensal e nunca. A partir das informações geradas por esse instrumento, foi delineado o perfil inicial de consumo, para embasar a avaliação das mudanças alimentares que porventura ocorressem ao longo das 40 semanas de intervenção.

Foi proposta uma metodologia utilizando o cômputo geral da freqüência de consumo, categorizado de modo que todas as freqüências foram transformadas em frações da freqüência diária, ou seja, na proporção de 30/30, equivalente a 1. Nesse enfoque, um consumo de 5 vezes na semana foi transformado em $20 / 30$ ou 0,$67 ; 4$ vezes na semana em $16 / 30$ ou 0,53 e assim sucessivamente, até a freqüência zero representada pela opção "nunca". A partir daí, calculou-se a média ponderada de freqüência de consumo para cada item e em seguida foi proposto o seguinte ponto de corte para classificação dos alimentos: 1 a 0,66 (alimentos de consumo expressivo); 0,65 a 0,33 (alimentos de consumo médio) e 0,32 a 0 (alimentos de consumo inexpressivo).

Nesse sentido, os alimentos classificados como de consumo expressivo e de consumo médio foram empregados na construção do perfil alimentar dos grupos nos momentos pré e pós-intervenção.

Com o objetivo de explorar com mais detalhes as informações referentes ao consumo alimentar, os itens do questionário que tiveram consumo expressivo e médio foram em seguida organizados em 9 grupos de alimentos: Pães, 
cereais, raízes e tubérculos (pães, farinhas, massas, bolos, biscoitos, cereais matinais, arroz, feculentos e tubérculos); Hortaliças (todas as verduras e legumes com exceção das citadas no grupo anterior); Frutas (cítricas e não cítricas); Carnes (carne bovina e suína, aves, peixes, ovos, miúdos e vísceras); Leite (leites, queijos e iogurtes); Leguminosas (feijão, soja, ervilha, grão de bico, fava e amendoim); Óleos e gorduras (margarina/ manteiga, óleo, maionese e creme de leite); Açúcares e doces (doces, mel, açúcares e adoçante artificial); Bebidas e infusões (café, sucos, refrigerantes e chás).

Os alimentos típicos da região foram analisados em separado. Finalmente, a avaliação do consumo alimentar incluiu ainda questões qualitativas referentes a alguns alimentos, como: tipo de óleo, de margarina e de leite utilizados; espécie de peixe, de frutas e de sucos de frutas preferidos.

A intervenção nutricional foi inteiramente conduzida por nutricionista, que na consulta inicial aplicou um questionário para levantamento de informações básicas, necessárias à caracterização da população e ao cálculo da dieta. Após essa consulta, os indivíduos foram orientados a cumprir dieta hipocalórica, calculada individualmente e obedecendo, quando possível, às preferências e hábitos previamente relatados.

O cálculo da dieta foi feito com base no aporte energético diário de $25 \mathrm{kcal} / \mathrm{kg}$ de peso desejado7, calculado pelo IMC médio da faixa anterior de classificação do risco e manejo da obesidade atual, proposta pela Primeira Convenção Latino-Americana para Consenso em Obesidade 1 , sendo as calorias oriundas de carboidratos (55\%-60\%), lipídeos (20\%-25\%) e proteínas (15\%-20\%) distribuídas em 5 ou 6 refeições/dia, contemplando ainda, aumento no aporte de fibras e redução na ingestão de gorduras saturadas, colesterol, carboidratos simples e sódio.

O plano dietético entregue a cada indivíduo, foi constituído de um cardápio básico fixo, com grupos de alimentos e porções, distribuídos entre as refeições, com seus respectivos horários e lista de substituições de alimentos equivalentes, conforme proposto por Tuma e Monteiro ${ }^{8}$. Incluiram-se orientações relativas à escolha de ingredientes, forma de preparo dos alimentos e hábitos saudáveis de alimentação e de vida.

Nas quatro consultas subseqüentes, avaliaram-se os resultados parciais da variação do peso, para, quando necessário, reajustar os cálculos do Valor Energético Total da dieta. Nesses contatos, discutiam-se as eventuais dificuldades encontradas no cumprimento do plano dietético, para assim melhorar a adesão e ampliar as chances de se alcançar os objetivos propostos.

Nos encontros de nutrição, eram abordados temas referentes à alimentação, nutrição e saúde, enfatizando sempre a relação entre a adoção de hábitos alimentares saudáveis e a melhoria da qualidade de vida. Além disso, tais reuniões propiciavam um espaço para socialização, trocas de experiência e esclarecimentos de dúvidas referentes ao plano dietético, configurando assim um trabalho de educação alimentar.

A atividade física foi aplicada somente para GE, por professor de educação física; acompanhou-se a freqüência cardíaca ( $F C$ ) individualmente por meio de monitor de freqüência cardíaca da marca Polar, durante todo o exercício, para assegurar sua manutenção dentro da faixa preconizada. Aplicou-se um protocolo constituído de exercício aeróbio do tipo caminhada em quadra coberta: 5 minutos de aquecimento, 45 minutos de caminhada (60\% a $80 \%$ da FC máxima) e 10 minutos de alongamento. O GD foi orientado a manter seu padrão inicial de atividade física (sedentário).

Na tabulação e análise dos dados foram utilizados os programas Epi Info versão 6.04b e Excel97. Para os dados categorizados, utilizou-se o Teste Exato de Fisher e o Teste Qui-quadrado de Pearson, com uma significância de 5\% $(\alpha=0,05)$, fixada também para a comparação das médias, aplicando-se para isso o teste Não Paramétrico de Mann-Whitney. 


\section{RES ULTA D O S}

Do universo inicial de 30 indivíduos, houve uma perda de 13,3\%, por desligamento espontâneo do programa, passando a constituir a amostra um total de 26 indivíduos: 13 para o Grupo Dieta (GD) e 13 para o Grupo Exercício (GE). A idade média dos grupos estudados foi de 61,1 anos (GD) e 55,5 anos (GE). Quanto à escolaridade, a distribuição de ambos os grupos foi semelhante, tendo a maioria $(46,1 \%)$ apenas o Ensino Fundamental incompleto.

A análise comparativa dos parâmetros antropométricos iniciais, demonstrou uma distribuição homogênea entre os grupos estudados, apresentando o GD: peso corporal médio de 78,8kg; $I M C=33,77$; relação $C / Q$ 0,94cm; circunferência da cintura (CC) $103,69 \mathrm{~cm}$. O GE apresentou: peso corporal médio de $84,1 \mathrm{~kg}$; IMC=35,91; relação C/Q 0,94cm; circunferência da cintura (CC) $106,50 \mathrm{~cm}$.

Em relação à evolução da variável peso corporal ao longo do estudo, foi constatado que, apesar dos grupos apresentarem perda média de $2,3 \mathrm{~kg}(\mathrm{GD})$ e $5,3 \mathrm{~kg}(\mathrm{GE})$, tais mudanças não tiveram significância estatística ( $p$-valor 0,1062).

Entretanto, utilizando-se a classificação segundo o IMC, foi possível perceber que ocorreram mudanças positivas de categoria tanto no GD, como no GE (Tabela 1). A análise estatística demonstrou que houve diferença significativa, de $5 \%$ ( $p$-valor 0,0498), em relação às proporções de mudança positiva na classificação do IMC para ambos os grupos, sendo que o GE respondeu melhor ao tratamento.

A classificação obtida da relação cintura/ quadril (C/Q), apontou que $100 \%$ da amostra apresentava obesidade do tipo andróide pré e pós-tratamento, não havendo redução significativa deste parâmetro antropométrico em nenhum dos dois grupos ao final do estudo. Quanto à circunferência da cintura (CC), foi verificada uma tendência de redução desta medida após a intervenção, GD-4, $8 \mathrm{~cm}$, em especial para o $\mathrm{GE}-7,6 \mathrm{~cm}$.
Tabela 1. Distribuição do Grupo Dieta e Grupo Exercício segundo a classificação do Índice de Massa Corporal, pré e pós-intervenção.

\begin{tabular}{lcccccc}
\hline & \multicolumn{2}{c}{ Grupo Dieta } & & \multicolumn{2}{c}{ Grupo Exercício } \\
\cline { 2 - 3 } \cline { 6 - 7 } & Pré & Pós & & Pré & Pós \\
\hline Peso saudável & 0 & 1 & & 0 & 0 \\
Risco moderado & 0 & 2 & & 0 & 3 \\
Risco alto & 10 & 7 & & 4 & 5 \\
Risco muito alto & 2 & 2 & & 7 & 5 \\
Risco extremo & 1 & 1 & & 2 & 0 \\
\hline Total & 13 & 13 & & 13 & 13 \\
\hline
\end{tabular}

Com relação ao padrão de refeições, ao final do estudo constatou-se que, apesar de terem sido prescritas entre 5 e 6 refeições diárias, em ambos os grupos, não houve modificação significativa no número de refeições referidas pelos indivíduos antes da intervenção. Em ordem decrescente, as refeições mais citadas foram: almoço, desjejum, jantar, colação e merenda.

No que se refere ao perfil alimentar dos grupos estudados, dos 58 itens contidos no questionário, menos da metade foi classificado como de "consumo médio e expressivo".

Do ponto de vista qualitativo, a alimentação referida por ambos os grupos durante as entrevistas, permitiu inferir que os mesmos apresentavam perfil de consumo bastante semelhante entre si. Isso justificou a análise unificada do consumo de todos os indivíduos, utilizando-se a categorização por grupos de alimentos, com acompanhamento das mudanças ocorridas ao longo do estudo.

1. Grupo de pães, cereais, raízes e tubérculos: os itens mais consumidos, em ordem decrescente, foram arroz, pão, farinha de mandioca, bolacha e torrada. Massas, feculentos e tubérculos não tiveram consumo expressivo. Não houve mudança significativa ao final da intervenção.

2. Grupo das hortaliças: no início do estudo foi mencionado apenas o consumo de repolho; após 40 semanas de intervenção, outros 6 tipos 
de hortaliças (alface, maxixe, pepino, tomate, abóbora e cenoura) passaram a ter consumo médio e expressivo.

3. Grupo das frutas: nos dois momentos do estudo, foi referido o consumo de banana, maçã e laranja, apesar de terem sido citados cerca de 15 tipos diferentes de frutas, inclusive as regionais como jenipapo, carambola e cupuaçú.

4. Grupo das carnes: antes da intervenção havia maior preferência por frango, seguido de carne de boi e peixe. Após a intervenção, apesar do frango se manter como de maior preferência, o consumo de peixe foi superior ao de carne. Foi inexpressivo o consumo de vísceras, embutidos e ovos. Os peixes mais citados, tanto no momento pré como no pós-intervenção foram o jaraqui (gordo), o tambaqui (semigordos) e o tucunaré (magro). O uso de peixes magros na alimentação, apesar de ter sido incentivado ao longo do trabalho, aumentou apenas $14 \%$.

5. Grupo do leite: foi constatado um consumo bastante expressivo de leite, nos momento pré e pós-intervenção; porém, derivados como o iogurte e os queijos em geral, não fizeram parte do perfil alimentar dos indivíduos estudados. Do ponto de vista qualitativo, houve mudança no tipo de leite consumido, com aumento de cerca de $30 \%$ na preferência por leite desnatado.

6. Grupo das leguminosas: representado exclusivamente pelo feijão, sendo considerado de consumo expressivo pré e pós-intervenção.

7. Grupo dos óleos e gorduras: durante o estudo, o óleo e a margarina tiveram consumo expressivo pré e pós-intervenção, ao contrário da manteiga, creme de leite e maionese que foram consumidos de forma inexpressiva. Do ponto de vista qualitativo, pode-se perceber que, apesar do óleo de soja ter sido referido pela maioria $(63,0 \%)$, já se encontra também o consumo de óleo de milho (29,6\%). Em relação ao tipo de margarina, ao final do estudo observou-se aumento na ordem de 40,0\% na preferência por halvarina.

8. Grupo dos açúcares e doces: no período pré-intervenção houve consumo expressivo de açúcar, que após a intervenção foi substituído por adoçante artificial.

9. Grupo das bebidas e infusões: foi observada presença marcante dos itens café, suco de frutas e chá, tanto no início como ao final da intervenção. Os refrigerantes não fizeram parte do perfil alimentar dos indivíduos deste estudo. Importa ressaltar que, dos 19 tipos de sucos referidos pelos indivíduos, 94\% eram sucos de frutas naturais, com preferência pelos de acerola, abacaxi e carambola, nesta ordem.

Dos alimentos típicos da região, somente a macaxeira e a tapioquinha apresentaram discreto destaque; os demais itens desse grupo tiveram freqüência de consumo pouco expressiva, mesmo após as 40 semanas de intervenção.

\section{I S C U S S Ã O}

O presente estudo apresentou perda amostral de 13,3\%, considerada baixa para uma intervenção de 40 semanas, se comparado a estudo semelhante também realizado com mulheres obesas pós-menopausa9.

Foi possível constatar, que as reduções ponderais verificadas no presente estudo para o GD e para o GE estão coerentes com aquelas encontradas por outros autores em trabalhos semelhantes ${ }^{10}$.

A evolução do IMC ao longo do estudo, mostrou-se reveladora da importância do programa proposto, pois, ficou constatado, que houve mudanças positivas de categoria na classificação, e que estas foram mais marcantes para o GE; isto responde, portanto, a uma expectativa mais realista de reduzir a intensidade dos riscos para a saúde dos indivíduos, por meio de exercício físico e acompanhamento nutricional. Esta expectativa foi justamente utilizada como base para o cálculo individual de cada dieta. Infelizmente, na literatura consultada, não se encontrou nenhum estudo que permitisse qualquer comparação com a referida classificação do IMC. 
Em relação à distribuiç̧ão de gordura corporal, houve predominância de acúmulo na região abdominal, retratando um perfil de obesidade bastante documentado em mulheres no climatério, o que pode ser devido às alterações hormonais impostas por esta fase ${ }^{11}$. Ao longo do estudo, a relação cintura/quadril não sofreu mudanças e, apesar de ter havido uma tendência de redução da circunferência da cintura para o GE, o programa proposto não foi capaz de reduzir significativamente essas medidas, conforme também observado por Stefanick et al. ${ }^{10}$, em pesquisas com mulheres no período do climatério.

O objetivo maior de qualquer proposta terapêutica para a obesidade deve ser realista e maleável, levando em conta uma duração indeterminada, especialmente para o grupo populacional em questão ${ }^{4}$. Torna-se necessário, muito mais do que avaliar perdas de peso isoladas, avaliar redução de riscos para a saúde, promovendo mudanças de comportamento e, conseqüentemente, melhoria na qualidade de vida. Nesse sentido, a evolução observada nas 40 semanas de intervenção, possibilitou analisar o efeito do programa proposto sobre a melhoria do perfil antropométrico dos participantes; foi possível constatar que, embora a magnitude das mudanças não tenha sido grande, a sua eficiência tornou-se clara após a interpretação devida dos resultados.

A análise do tipo de alimentação dos indivíduos de ambos os grupos apontou um consumo de características monótonas, contando apenas com cerca de 20 itens de "consumo médio e expressivo", para composição do perfil alimentar. Entretanto, após as 40 semanas de aconselhamento nutricional, algumas mudanças importantes foram verificadas.

No grupo das hortaliças foi evidenciada a inclusão de 6 novos itens (alface, maxixe, pepino, tomate, abóbora e cenoura) no padrão alimentar. Resultado bastante interessante, pois as informações disponíveis sobre a população de Manaus, embora datem da década de 70 , referem que a quantidade consumida de hortaliças era baixíssima, havendo mais uso destas somente como tempero ${ }^{12}$. Em âmbito nacional, pesquisas realizadas pelo IBOPE, com 6 mil famílias, verificaram que apenas $58 \%$ dos brasileiros referem o consumo de legumes ${ }^{13}$. Na realidade, o uso de vegetais em algumas regiões do país é reduzido, por uma questão cultural, pois os índios brasileiros não davam importância a tais alimentos e sua introdução na nossa culinária sofreu influência dos negros escravizados ${ }^{14}$.

A inclusão de novos itens do grupo das hortaliças no hábito alimentar de indivíduos obesos incrementa o aporte de fibras, as quais desempenham importantes funções na dieta para redução de peso, tais como: redução na ingestão energética, aumento no tempo de esvaziamento gástrico, redução na secreção de insulina, aumento na sensação de saciedade, diminuição na digestibilidade e aumento na excreção fecal de energia ${ }^{15}$.

Dietas ricas em fibras parecem minimizar os problemas de doenças cardiovasculares, devido à redução do colesterol total e da LDL plasmáticos, interferindo na excreção fecal de colesterol ligado aos ácidos biliares ${ }^{15}$. Em adição, as hortaliças constituem fontes clássicas de vitaminas e minerais. Tais informações, sobre os benefícios das fibras para o bom funcionamento orgânico, constituíram tema freqüente de discussão nos encontros de nutrição e, ao longo da pesquisa, estimulou-se o consumo de vegetais nas refeições, especialmente sob a forma de saladas cruas.

No grupo das carnes, constatou-se aumento no consumo de peixes, embora nem sempre de peixes magros, e redução no consumo de carne bovina. Isto está de acordo com a tendência nacional de substituir-se das carnes ditas "vermelhas" (bovina e suína), por carnes "brancas" (aves e pescados), principalmente quando as pessoas passam a conhecer melhor os alimentos e a preocupar-se com a qualidade de sua alimentação ${ }^{16}$.

Um detalhe peculiar foi observado em relação ao tipo de peixe consumido, pois a preferência por jaraqui e tambaqui parece resistir às mudanças dos tempos: também foi referida 
em trabalho de consumo alimentar, realizado na mesma região há décadas atrás ${ }^{12}$.

No grupo do leite, a maior alteração observada foi a substituição do leite integral pelo desnatado. Esta excelente escolha, provavelmente foi pautada nos conhecimentos adquiridos nos encontros de nutrição, ocasião em que se orientava os participantes sobre a correta substituição de certos alimentos por similares mais saudáveis e de custo acessível. Neste caso, o leite desnatado contribuiu para a redução da ingestão de gordura e colesterol, sem interferir no aporte de cálcio, tão necessário como fator protetor contra a osteoporose, para mulheres no climatério.

No grupo dos óleos e gorduras verificou-se o aumento na preferência por halvarina e óleos de soja, de milho e de girassol. A introdução de halvarina no lugar da margarina ou da manteiga, também refletiu conhecimentos adquiridos ao longo do programa, referentes às boas escolhas; neste caso, no sentido de reduzir o consumo de gordura saturada e colesterol. O emprego de óleos vegetais de diversas origens no preparo dos alimentos, é uma tendência já observada por Sichieri ${ }^{11}$, especialmente entre indivíduos de idade mais avançada.

No grupo dos açúcares, percebeu-se marcada substituição do açúcar por adoçante artificial, demonstrando assim mais uma vez, que os indivíduos foram mobilizados em relação às mudanças desejadas para, não só reduzir o peso corporal, como também construir novos hábitos alimentares. A oferta deste tipo de produto e de outros da linha diet/light, parece estar crescendo no nosso país, disseminando seu uso entre as diversas camadas da população ${ }^{11}$.

Importa ressaltar a presença considerável de frutas e de sucos naturais diversos, no perfil alimentar tanto do início como do final do experimento; este comportamento opõe-se aos observados por alguns autores, que reportam baixo consumo desses alimentos entre indivíduos obesos ${ }^{17}$. Segundo Bleil ${ }^{13}$, pesquisas indicam que somente $44 \%$ dos brasileiros relatam o consumo de frutas. Por outro lado, Najas et al. ${ }^{18}$, num trabalho de caracterização do padrão alimentar de idosos de diferentes classes socioeconômicas na região sudeste, evidenciou consumo expressivo de frutas, levantando a hipótese de que indivíduos dessa faixa etária valorizam o grupo dos alimentos reguladores.

Com isso, ficou destacado que a associação de dieta individualizada e educação nutricional em grupo foi bem sucedida no sentido de modular o padrão dietético dos indivíduos, contribuindo para amenizar o consumo excessivo de gorduras, colesterol e carboidratos simples, que comprovadamente possuem ação deletéria sobre o organismo, prejudicando o balanço energético e o metabolismo oxidativo ${ }^{15}$.

Em relação aos alimentos típicos da região, o baixo consumo verificado no presente estudo, parece endossar um fenômeno mais amplo de incorporação de hábitos alimentares importados. Nas últimas décadas, a população vem reduzindo o consumo de produtos tradicionais, como a macaxeira, o cará e outros tipos de raízes, que outrora representaram o principal alimento do nativo brasileiro ${ }^{13}$.

O padrão alimentar da população urbana no Brasil, sem dúvida, está progressivamente absorvendo as novidades criadas pela indústria, tornando-se cada vez mais homogêneo. Num mundo globalizado, isto parece inevitável, gerando graves conseqüências para a saúde pública, associadas ao aumento das doenças crônicas e aos desvios ponderais ${ }^{13}$.

Neste panorama de consumo uniformizado e construído pela mídia, torna-se um desafio a atuação do nutricionista como educador, principalmente se for considerada toda a complexidade que envolve a seleção individual de alimentos ${ }^{14}$. Segundo Chapman et al. apud Assis \& Nahas"19, existe uma relação "altamente tênue" entre o que as pessoas sabem e o que as pessoas fazem. O conhecimento não instiga a mudança, mas pode funcionar como um instrumento quando as pessoas desejam mudar.

O sucesso da intervenção nutricional parece depender do entendimento dos papéis 
desempenhado pelos alimentos na vida das pessoas; estes, muito mais do que meras fontes de nutrientes para a sobrevivência, são fontes de gratificações emocionais e meios de expressar valores e relações sociais ${ }^{20}$. Isto torna vantajosa a análise de alimentos ou de grupos de alimentos, em detrimento da análise de nutrientes, pois possibilita avaliar o comportamento alimentar e assim propor, mais facilmente, modificações no consumo, em busca de hábitos de vida saudáveis.

No presente estudo, a intervenção nutricional proposta operou mudanças importantes nos padrões alimentares de mulheres obesas no climatério; isto corrobora a posição dos especialistas a respeito da aplicação de técnicas de mudança do comportamento, para eles, esta mudança constitue, o terceiro componente fundamental, junto com o exercício físico e dieta, para o sucesso de programas de combate à obesidade? .

\section{O N C L U S Ã O}

Nas condições do presente estudo, a análise dos resultados obtidos com a aplicação de um programa de intervenção nutricional e de exercício físico sobre o perfil antropométrico e os hábitos alimentares de mulheres obesas no climatério, permitiu concluir que:

- A perda de peso corporal dá-se com maior intensidade na presença de exercício físico.

- A associação de dieta e educação nutricional mostrou-se efetiva na promoção de mudanças no consumo habitual de alimentos.

\section{RE FER Ê N C I A S}

1. Primeiro Consenso Latino-Americano em Obesidade. $1^{\text {a }}$ Convenção Latino-Americana para consenso em obesidade. Rio de Janeiro: Ministério da Saúde do Brasil; 1998.

2. World Health Organization. Report of a WHO consultation on obesity. Obesity - preventing and managing the global epidemic. Geneva; 1997.
3. Milewicz A, Bidzinska B, Sindorowicz A. Perimenopausal obesity. Gynecol Endocrinol 1996; 10:285-91.

4. Mundim ER. Obesidade: reflexões. Rev Med Minas Gerais 1996; 6(1):20-5.

5. Montilla RNG. Avaliação do estado nutricional e do consumo alimentar de mulheres no climatério de um Centro de Saúde-Escola [dissertação]. São Paulo: Universidade de São Paulo; 2001.

6. Rogers PJ. Eating habits and appetite control: A psychobiological perspective. Proc Nutr Soc 1998; 58(1):59-67.

7. Guedes DP, Guedes JER. Controle do peso corporal: composição corporal, atividade física e nutrição. Londrina: Midiograf; 1998. 312p.

8. Tuma R, Monteiro R. Tabela de Alimentos equivalentes Nutrivisa. Manaus: 1999. 59p.

9. Thompson JL, Butterfield GE, Jylfadothir UK. Effect of human growth hormone, insulin-like growth factor I, and diet and exercise on body composition of obese postmenopausal women. J Clin Endocrinol Metab 1998; 83(5):1477-84.

10. Stefanick ML, et al. Effect of diet and exercise in men and postmenopausal women with low levels of HDL cholesterol and high level of LDL cholesterol. N Engl J Med 1998; 339:12-20.

11. Sichieri R. Epidemiologia da obesidade. Rio de Janeiro: EdEURJ; 1998. 140p.

12. Giugliano R, Shrimpton R, Arkcoll DB, Giugliano LG, Petrere Jr M. Diagnóstico da realidade alimentar e nutricional do Estado do Amazonas. Rev Acta Amazônica 1978; 8(2 Supl 2):36-41.

13. Bleil SI. O Padrão alimentar ocidental: considerações sobre a mudança de hábitos no Brasil. Cad Debate 1998; 6:1-25.

14. Ramalho RA, Saunders C. O papel da educação nutricional no combate às carências nutricionais. Rev Nutr 2000; 13(1)11-6.

15. Francischi RPP, Lancha Jünior AH, Pereira LO, Freitas CS, Klopfer M, Santos RC, et al. Obesidade: atualização sobre sua etiologia, morbidade e tratamento. Rev Nutr 2000; 13(1):17-28. 
16. Instituto Brasileiro de Geografia e Estatística. Pesquisa de Orçamentos Familiares 1995/1996. Consumo Alimentar Domiciliar per capita. Rio de Janeiro: IBGE; 1996. Capítulo 2:27-31.

17. Angelis RC. Novos conceitos em nutrição: reflexões a respeito do elo dieta e saúde. Arq Gastroenterol 2001; 38(4):269-71.

18. Najas MS, Andreazja R, Souza ALM, Sachs A, Guedes LRS, Ramos LR, et al. Padrão alimentar de idosos de diferentes estratos socioeconômicos residentes em localidade urbana da região sudeste, Brasil. Rev Saude Publica 1994; 28(3):187-91.

19. Assis MAA, Nahas MV. Aspectos motivacionais em programas de mudança de comportamento alimentar. Rev Nutr 1999; 12(1):33-41.

20. Casotti L, Ribeiro A, Santos C, Ribeiro P. Consumo de alimentos e nutrição: dificuldades práticas e teóricas. Cad Debate 1998; 6:26-39.

Recebido para publicação em 25 de julho de 2002 e aceito em 6 de novembro de 2003. 